\section{ISCB Afirica ASBCB Conference on Bioinformatics and eBioKit Workshop a}
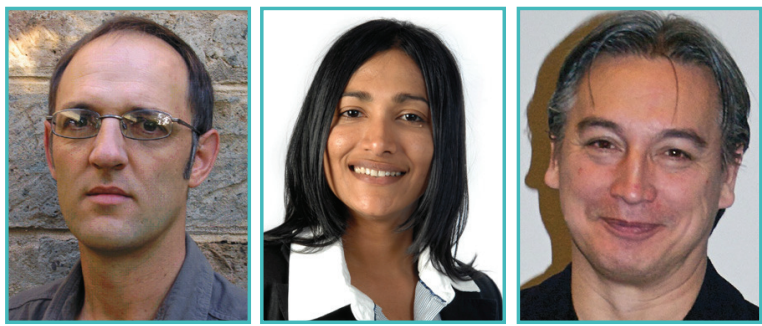

Etienne de Villiers', Judit Kumuthini ${ }^{2}$, Erik Bongcam-Rudloff ${ }^{3}$

'ILRI Bioinformatics group, Kenya

${ }^{2}$ Centre for Proteomic and Genomic Research (CPGR), South Africa

${ }^{3}$ Swedish University of Agricultural Sciences, Uppsala, Sweden

The International Society for Computational Biology $\left(\underline{\left.\mid S^{\prime} B^{\prime}\right)}\right.$ ) and the African Society for Bioinformatics and Computational Biology $\left(\mathrm{ASBCB}^{2}\right)$ held the ISCB Africa ASBCB Conference on Bioinformatics in Cape Town, South Africa, in March 2011. The meeting constituted the second joint meeting of ISCB and $A S B C B$, and the third conference of the ASBCB on the Bioinformatics of African Pathogens, Hosts and Vectors. The conference was preceded by a two-day workshop at the University of the Western Cape ${ }^{3}$. ASBCB is a society dedicated to the advancement of bioinformatics and computational biology in Africa.

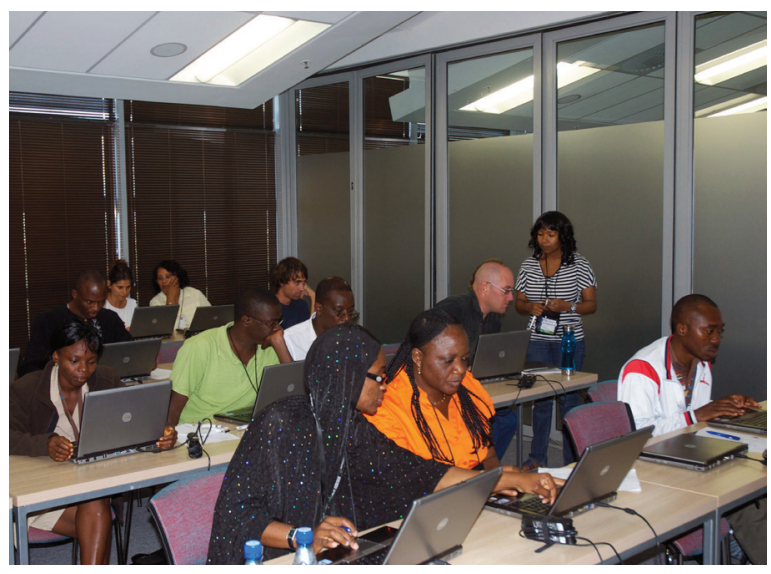

Participants at the eBioKit tutorials.

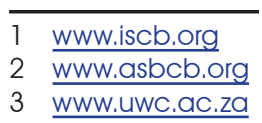

The society works with the ISCB African Regional Student Groups ${ }^{4}$ to provide training courses and a mentorship programme, to help train the current and next generation of African bioinformatics students.

EMBnet was very well represented at the conference, both as one of the official sponsors, and in providing two trainers for the preceding workshop. EMBnet had an exhibition stand with promotional material, including copies of EMBnet. journal and its new promotional pamphlet, organised by Judit Kumuthini from the Centre for Proteomic and Genomic Research (CPGR5), and the EMBnet node in South Africa. Displayed for the first time was a promotional poster that is now being presented at all major meetings, courtesy of EMBnet's Publicity \& Public Relations Project Committee.

Many visitors to the EMBnet stand were interested in signing up to the mailing list, indicating the continuing interest of scientists in Africa in EMBnet's activities. Several also expressed interest in establishing a dedicated node for their country or Institute.

At the stand, there was a working copy of the eBioKit ${ }^{\circ}$, developed by the research team of Erik Bongcam-Rudloff, which was very well received by attendees. eBioKit is a novel system for teaching bioinformatics in places where there is limited Internet access, and hence limited online access to bioinformatics software and databases. Many of these resources are installed

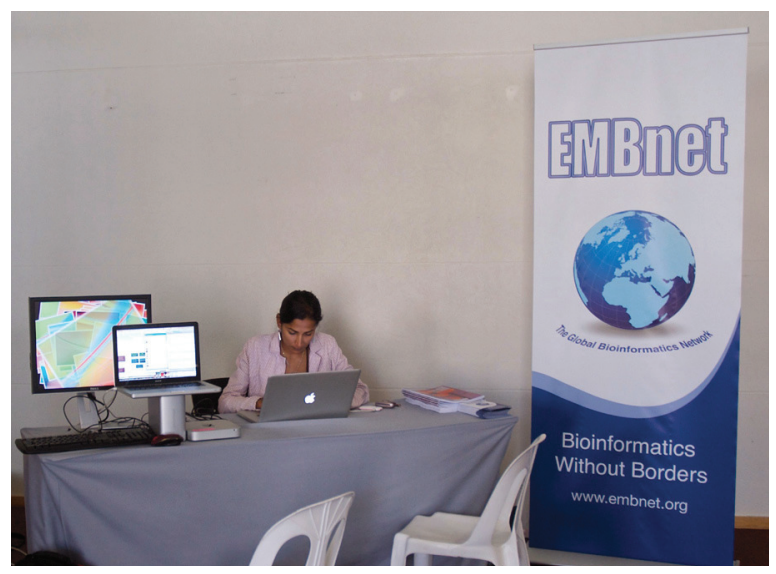

EMBnet stand.

4 www.iscbsc.org/content/regional-student-groups

5 www.cpgr.org.za

6 http://collab.hgen.slu.se/software/ebiokit 


\section{REPORTS EMBnet.jourmal 17 Mr.2}

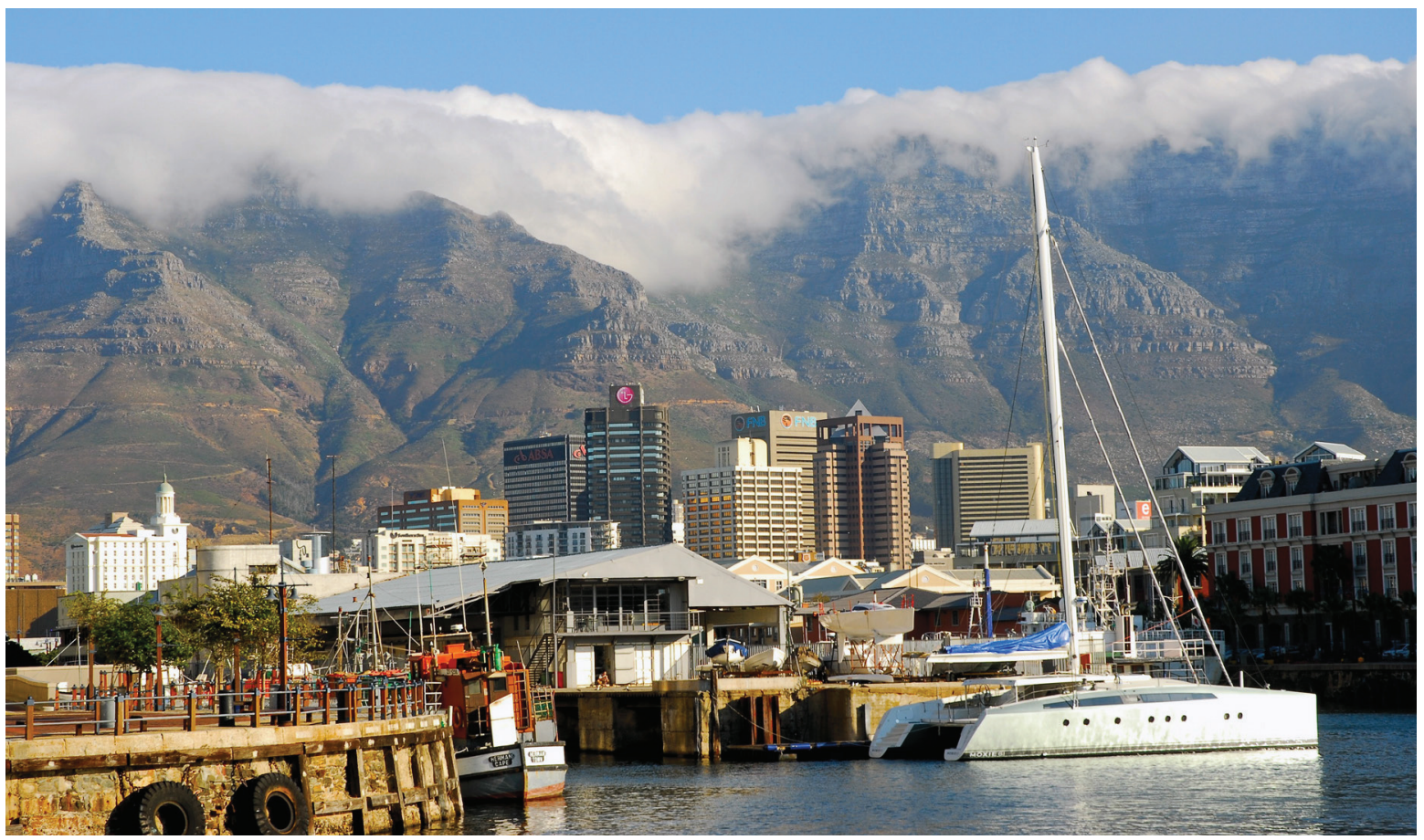

Cape Town.

on the eBiokit by default, including Ensembl'. One of the co-developers of Jalview $2^{8}$, Dr. David Martin from Dundee University?', Scotland, kindly offered to install Jalview, which is therefore now also available as part of the eBiokit package.

The two-day workshop was held at SANBI (South African Bioinformatics Institute ${ }^{10}$ ), University of Western Cape $\left(\underline{U W C}^{\prime \prime}\right)$ in Cape Town, South Africa, on the $7^{\text {th }}$ and $8^{\text {th }}$ of March, with three parallel sessions: the first showcased the online tools of the European Bioinformatics Institute $\left(\underline{\underline{E B}}{ }^{12}\right)$; the second introduced EMBnet's eBioKit; and the third concerned Genome Wide Association Studies (GWAS) and population genetics.

The EBI Roadshow workshop included sessions that introduced participants to databases and tools hosted at EBI, including those for sequence searching and alignment, gene expression data analysis, and interaction and pathway analysis. These popular sessions attracted 30 or more participants.

Erik Bongcam-Rudloff from the Swedish EMBnet node, Etienne de Villiers from the Kenyan

\footnotetext{
7 www.ensembl.org

8 www.jalview.org

9 www.dundee.ac.uk

10 www.sanbi.ac.za

11 www.uwc.ac.za

12 www.ebi.ac.uk
}

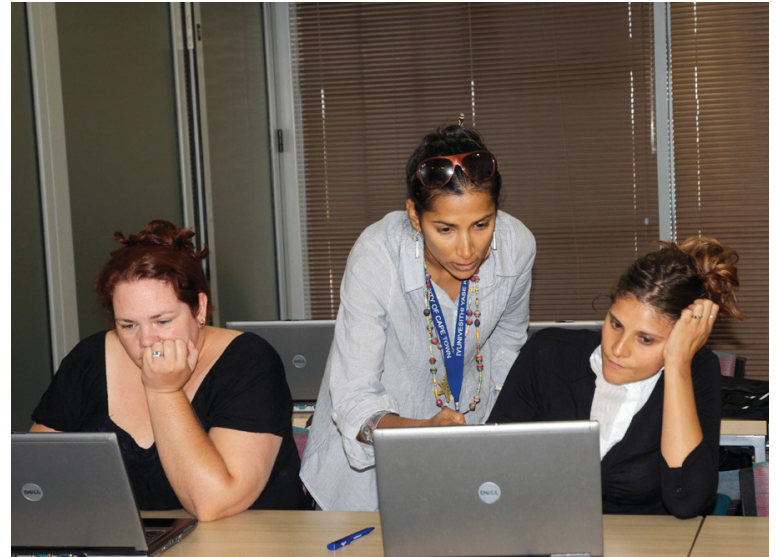

Judith Kumuthini supervising students.

EMBnet node ${ }^{13}$, and Judit Kumuthini and Dane Kennedy from CPGR, taught the eBioKit workshop. This attracted around 20 participants from a variety of Institutions and Universities of several different African countries, including biologists, computational biologists, geneticists and bioinformaticians. The main objectives were to: introduce the eBioKit, with its large set of commonly used bioinformatics tools and databases, and promote its use as an advanced training tool; promote the participation and training of new in-

13 http://hpc.ilri.cgiar.org 


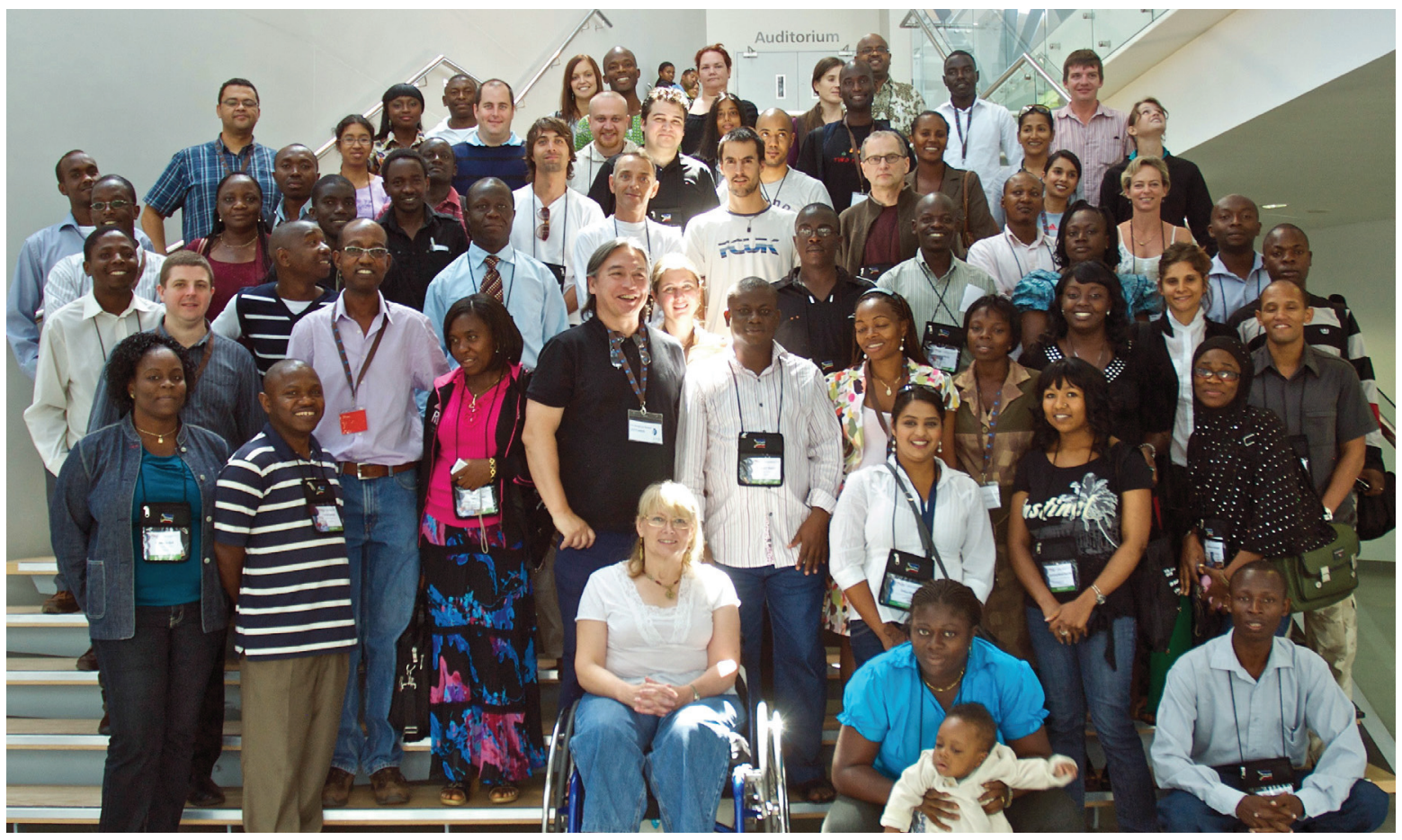

Workshop participants.

vestigators in the field of bioinformatics; promote communication between these scientists and locally relevant bioinformatics efforts through EMBnet's activities; and strengthen the bioinformatics network in Africa via EMBnet.

Following the introduction to the eBiokit were sessions demonstrating both how to access its installed databases using MRS ${ }^{14}$, and how to use EMBOSS/wEMBOSS (Sarachu and Colet, 2004). Judit Kumuthini and Dane Kennedy were on hand to give help during the practical demonstrations. On the second day, participants were introduced to two more resources found in the eBioKit, namely Ensembl and Galaxy ${ }^{15}$, a Webbased platform for data-intensive biomedical research.

Now a global bioinformatics network, part of EMBnet's mission is to foster bioinformatics training and to disseminate bioinformatics skills throughout its member countries. This was the first time that EMBnet officially sponsored an event not directly related to its member activities, but was an important opportunity to synergise with the work of the ASBCB, one that we hope to embrace again in future. In his closing speech,
Daniel Massiga, Chair of the ASBCB, warmly recognised EMBnet's role in this collaborative approach to developing education and skills in the African continent.

\section{References}

Sarachu M, Colet M (2004) wEMBOSS: a web interface for EMBOSS. Bioinformatics 21, 540-541. 